Bond University

Research Repository

\title{
Constraints to Leveraging Regular Season Sport Team Events
}

Sobral, Vitor; Fairley, Sheranne; O'Brien, Danny

Published in:

Sport Management Review

DOI:

10.1080/14413523.2021.1989238

Licence:

Other

Link to output in Bond University research repository.

Recommended citation(APA):

Sobral, V., Fairley, S., \& O'Brien, D. (2021). Constraints to Leveraging Regular Season Sport Team Events. Sport Management Review. https://doi.org/10.1080/14413523.2021.1989238

\footnotetext{
General rights

Copyright and moral rights for the publications made accessible in the public portal are retained by the authors and/or other copyright owners and it is a condition of accessing publications that users recognise and abide by the legal requirements associated with these rights.
}

For more information, or if you believe that this document breaches copyright, please contact the Bond University research repository coordinator. 
Constraints to Leveraging Regular Season Sport Team Events for Tourism Benefits 


\title{
Constraints to Leveraging Regular Season Sport Team Events for Tourism Benefits
}

Vitor Sobral (corresponding author)

The University of Queensland, Room 455, General Purpose North 3 (39A), St Lucia Campus, QLD, Australia

v.sobral@uq.edu.au

\begin{abstract}
Research on event leveraging has revealed that certain constraints inhibit the realisation of desired benefits from event hosting. Using qualitative action research methods, this study examined the constraints to leveraging regular season professional sport team events for tourism. The leveraging potential of regular season professional sport team events has been largely ignored - both by researchers and practitioners. This presented an ideal opportunity to examine the pre-leveraging phase with a view to understanding leveraging constraints. The results identified five overarching constraints: lack of collaboration; priorities and resources; perceived benefit radius; perceived tourism potential; and, unclaimed responsibility. We propose a process model to better understand how leveraging constraints emerge and can be negotiated over time. This research is among the first to demonstrate the utility of team events for inclusion in regional event portfolios. It, therefore, builds on extant knowledge by presenting a more holistic conceptualisation of the inherent constraints to event leveraging, and further, provides a basis from which to successfully negotiate these constraints.
\end{abstract}

\section{Keywords}

event leverage; professional sport team events; sport tourism; event tourism; constraints; action research 


\section{Introduction}

As pressure for events to generate economic, social, and other benefits for host destinations has grown (Chalip, 2017; Karadakis, Kaplanidou, \& Karlis, 2010), so too has the practice of strategic event leveraging (Chalip, 2017; Kelly \& Fairley, 2018). From an event leveraging perspective, a portfolio of events is a leverageable resource that creates opportunities which, if specific strategies are implemented, can lead to targeted benefits for the host community (Chalip, 2004). Events have been leveraged to achieve a range of objectives, including economic outcomes (O’Brien \& Gardiner, 2006), image enhancement (Grix, 2012), and social benefits (O’Brien \& Chalip, 2007). Since economic objectives through tourism are a significant reason for leveraging events (Kelly \& Fairley, 2018), this research considers the leveraging of regular season sport events for tourism benefits.

However, event leveraging can be subject to certain constraints (Bell \& Gallimore, 2015; Chalip, Green, Taks, \& Misener, 2017; Kennelly, Corbett, \& Toohey, 2017). As research to date has focused on one-off events, and despite Mason and Duquette's (2008) assertion that team events remain an untapped resource, current understanding of the constraints to leveraging regularly occurring events is limited. We address this shortcoming by examining the leverage of regular season professional sport team events for tourism benefits, specifically related to direct benefits of visitor spending, and indirect benefits of image enhancement (Chalip, 2004). Regular season professional sport team events will be referred to as 'team events' in this paper. Leveraging team events can be beneficial to host destinations as they generally take place within existing infrastructure and are, therefore, less financially costly and disruptive to the host society (Higham \& Hinch, 2003; Misener, Taks, Chalip, \& Green, 2015; Taks, Green, Misener, \& Chalip, 2014).

While previous research has identified different constraints to event leveraging, this study proposes a process model that identifies constraints in a previously unstudied event 
context and conceptualises the negotiation of these constraints. Team events have received relatively little leveraging attention and thus, provide a unique context to investigate event leveraging and constraints (Mason \& Duquette, 2008; Sparvero \& Chalip, 2007). Since team events are regularly occurring throughout any given year, there is no specific date for leveraging implementation, which adds another unique perspective to identifying constraints over time. The purpose of this study is to examine the constraints to leveraging team events for tourism benefit. The focus on constraints to leveraging team events, therefore, leads to a more holistic conceptualisation of constraints to leveraging wider event portfolios.

\section{Event Leveraging and Team Events}

Event leveraging is a strategic activity, as it conceptualises how to plan for and produce benefits from a strategic resource, in this case, an event or a region's portfolio of events (Chalip, 2004). Thus, event leveraging is related to the strategic planning process (Nieboer, 2011), which considers the actions required to achieve identified goals. From an event leveraging perspective, events are the 'seed capital' from which strategies can be formulated to produce targeted benefits for the host community (Chalip, 2017; O'Brien \& Chalip, 2007; O’Brien, 2006). Chalip's (2004) conceptualisation of event leverage suggests that a region's portfolio of events, coupled with each event's respective assets, constitutes a leverageable resource. Opportunities for leverage arise from the event portfolio through visitation and media attention, and hosts can formulate objectives and implement relevant means to take advantage of these opportunities to achieve identified community goals (Chalip, 2004, 2016, 2017). In effect, event leveraging takes an ex-ante view by identifying the desired benefits during the planning phase of the event, in contrast to the ex-post view which focuses on what eventuates after the fact from event hosting (Chalip, 2017; O'Brien \& Chalip, 2007).

Team events, either national or international, routinely attract visitation and media 4 
attention (Gibson, Willming, \& Holdnak, 2002, 2003; Higham, 1999). These types of events have been referred to as small-scale, given that they typically occur within existing infrastructure (Fairley, 2003; Gibson et al., 2003; Higham, 1999). However, some of these team events, such as those involving Spanish football team FC Barcelona, regularly attract between 50,000-100,000 attendees and a global viewing audience in the hundreds of millions (Xifra, 2009).

Team events attract outside visitors to the event's host destination (Fairley \& Gammon, 2005; Gibson, 1998; Gibson et al., 2002, 2003; Jones, 2008; Mason \& Duquette, 2008; Sparvero \& Chalip, 2007). These regular events provide host cities and regions with significant opportunities for leveraging both direct and indirect benefits (Gibson et al., 2002, 2003; Higham \& Hinch, 2003; Mason \& Duquette, 2008; Sparvero \& Chalip, 2007). However, there is little research on how team events can be leveraged to produce tourism benefits.

Since team events have not been widely recognised for leveraging, it is useful to consider their strategic use, or lack thereof, through the framework of strategic management. Within strategic management, the notion of strategic control refers to three key mechanisms: feedback controls (what happened and why?); concurrent controls (what is happening now in real time?); and, feedforward controls (what is likely to happen in the future?) (Harrison \& St. John, 2014; O’Brien, Parent, Ferkins, \& Gowthorp, 2019). Feedforward controls consist of two mechanisms - strategic surveillance and premise controls (Harrison \& St. John, 2014). Premise control refers to, "whether the information and assumptions used to plan current strategies and their related goals are still valid" (O'Brien et al., p. 138). The fact that team events have not been widely included in event portfolios or researched as leverageable assets for tourism development suggests an assumption that team events are either not leverageable, or not even seen as events. Thus, from a feedforward strategic control perspective, empirical 
research to establish whether such an assumption exists and is actually constraining leveraging activity, is warranted.

\subsection{Event Leveraging Constraints}

Research on event leveraging has identified a number of constraints that can inhibit the various stages of the leveraging process (Bell \& Gallimore, 2015; Kellett et al., 2008; Kennelly et al., 2017; Taks et al., 2014; Taks, Green, Misener, \& Chalip, 2018). In leisure studies, researchers have separated constraints on action from barriers that completely prevent action (Hinch, Jackson, Hudson, \& Walker, 2005; Lamont, Kennelly, \& Wilson, 2012). Constraints might include stakeholders' idiosyncratic needs and desires which, as inhibitors to action, can be negotiated through competing priorities (Lamont et al., 2012). In contrast, barriers are outright impediments to action. The focus of this research is on constraints to leverage and how these can be negotiated. In leveraging, constraints obviously present challenges to achieving desired event objectives (Chalip et al., 2017).

While researchers have identified constraints to leveraging one-off events, those identified to date have not been integrated into a process model that can facilitate the negotiation of leveraging constraints, and consequently, more effectively leverage events. Leveraging constraints previously proposed can be categorised as: collaboration, culture, capacity and resources, political issues, and responsibility.

\subsubsection{Collaboration}

Lack of collaboration has been identified as a constraint to leveraging in numerous studies (e.g., Chen \& Misener, 2019; Kennelly et al., 2017; Mhanna, Blake, \& Jones, 2017; Taks et al., 2018). For example, lack of collaborative planning and a failure to look beyond short-term outcomes constrained achieving leveraging benefits in the context of Sydney 2000 Olympic Games pre-event training camps (O’Brien \& Gardiner, 2006). O’Brien and Gardiner 
also identified the absence of a specific group responsible for leveraging, as illustrated by the disengagement of key positions immediately post-event, which prevented achievement of some longer-term goals.

Research attempting to formulate a model to successfully leverage for sport development goals recognised that the temporary nature of some leveraging organisations led to issues with ownership and knowledge transfer (Chalip et al., 2017). Equally, Mhanna et al. (2017) found the focus on event delivery, rather than on implementation of leveraging initiatives, was a significant constraint to leveraging tourism from the London 2012 Olympic Games. Mhanna et al. (2017) also noted a lack of effective collaboration contributed to a perception that the host area would not attract Games visitors because it lacked tourism attractions similar to those in the city centre.

Event owners' formal stipulations relating to supply chain characteristics can also be a constraint to leveraging for host communities (Kelly, Fairley \& O’Brien, 2019). In the context of the 2007 Cricket World Cup, the event owner's supplier and broadcast restrictions impeded achievement of economic benefits as well as the showcasing of local culture (Kelly et al., 2019). Similarly, Duignan, Down and O’Brien (2020) demonstrated how the regulation of Olympic space by 2016 Rio de Janeiro Olympic Games organisers initially precluded local business stakeholders from leveraging the Olympic opportunity.

Other major challenges arise in the form of non-event stakeholders' primary focus on achieving day-to-day priorities rather than developing leveraging initiatives, collaborative constraints relating to limitations from bid requirements, sponsor exclusivity, and lack of opportunities for local business (Chalip et al., 2017).

\subsubsection{Culture}

Cultural constraints in event leveraging may include influences stemming from 7 
national culture (Beesley \& Chalip, 2011), societal issues (Chen \& Misener, 2019) or organisational culture (Taks, et al., 2018). Research on the 2008 Beijing Olympic Games revealed how a different cultural understanding of event leverage profoundly impacted desired objectives (Beesley \& Chalip, 2011). The cultural aspect of hierarchy influenced a strong belief that Beijing, rather than greater China, should be the primary beneficiary, as the 2008 Olympic Games was 'their' event (Beesley \& Chalip, 2011).

Meanwhile, Leicestershire's attempt to leverage the London 2012 Olympic Games was constrained by a sense that it was it was London's event and therefore, the host city should be primary beneficiary (Chen \& Misener, 2019). This demonstrates how culture, both societal and organisational, can impact leveraging. Attitudes influenced by culture can also act as a leveraging constraint, particularly when based on a perception that outcomes would be 'automatically' achieved. In leveraging for sport development objectives, Taks et al. (2014) demonstrated how attitudes constrained leverage because of an 'insider' culture that held that sport participation would 'automatically' increase if the sport simply managed to get exposure (Misener, 2015; Taks, et al., 2018).

\subsubsection{Resources and Capacity}

Research by Hoskyn, Dickson, and Sotiriadou (2018) on leveraging an annual tennis event to increase participation found that sport clubs lacked the resources and capacity to integrate new participants gained as a result of leveraging initiatives. This extended to a lack of resources to implement planned strategies (Hoskyn et al., 2018). Similarly, a lack of resources was found to be a leveraging constraint in Cheshire's attempt to leverage the London 2012 Olympic Games as a non-host region (Bell \& Gallimore, 2015).

Prior work has identified resource deficits impede leveraging in two main ways: (1) inadequate staff numbers to manage leverage planning and activations; and, (2) insufficient 
physical infrastructure and personnel for integrating existing and newly recruited participants (Taks, et al., 2014; Clark \& Misener, 2015). Insufficient knowledge around the overall sport and event context, as well as management, marketing, grant attraction, and sponsorship solicitation have also been identified as capacity-based constraints (Chalip et al., 2017).

\subsubsection{Political Issues}

Changes in government can lead to shifts in political agendas and priorities (Chalip et al., 2017). Such shifts impact funding allocations that inevitably cascade through to events and leveraging initiatives (Bell \& Gallimore, 2015). Political constraints can emerge through an economic climate that differs from the one prevailing when the bid was won, leading to a change in objectives. Further, event steering groups or bid teams may fail to engage certain key stakeholders, possibly creating responsibility disconnects for leverage planning and implementation (Bell \& Gallimore, 2015). Bell and Gallimore (2015) and Chalip et al. (2017) showed that ineffective political structures can lead to poor collaboration, lack of common purpose, and how political structures may impede identification of responsibility for leveraging.

\subsubsection{Responsibility}

Inability to identify responsibility for leveraging coordination is typically founded on a common stakeholder assumption that 'someone else' is responsible (Misener, 2015). This 'someone else' clearly cannot be event organisers, whose focus is on event delivery, not leverage. Thus, as Chalip, et al (2017) noted, it is folly to hold event organisers responsible for leverage, particularly when it simply cannot be their focus and other event stakeholders have much more to gain from leveraging outcomes.

Kennelly et al. (2017) found that an inability to identify responsibility for leverage was a constraint at the Glasgow 2014 Commonwealth Games. They identified lack of 
leadership and leadership support, poor communication among stakeholders, and unhealthy levels of competition, created interrelated constraints to leverage. These constraints included unclear goals, imprecise planning, insufficient financial resources, competing priorities, and conflicting stakeholder agendas.

Interestingly, constraints relating to interorganisational competitiveness, ineffective collaboration and lack of resources were also identified in Taks and colleagues' (2018) research on leveraging for sport development goals. An inability to identify responsibility for leveraging remains a ubiquitous constraint to event leveraging (Chalip et al., 2017).

\subsection{Summary}

Several studies have established a range of constraints to event leveraging for targeted strategic outcomes. Ineffective collaboration among stakeholders (including common goal setting), ineffective leadership and responsibility identification, insufficient knowledge, resources, and capacity have each been empirically established as constraints to leverage. However, these studies have focused on one-off events that have already been identified for leveraging, with team events, despite their potential, receiving no attention.

Focusing on the leveraging opportunities of team events will expand current understanding by revealing constraints in the pre-event leveraging period, as well as over time, as team events are ongoing and have no end date. Further, considering events that are still in the planning and implementation phase can reveal constraints not previously considered. The recurring nature of regular season events presents the opportunity for organisational learning (Argyris \& Schon, 1978), allowing the anchoring and building of new knowledge. We focus our research on team events because, although this sector constitutes an almost ubiquitous aspect of the socioeconomic fabric of modern society, little to no empirical work has investigated this event domain from a leveraging perspective. More specifically, we 
ask, what are the constraints to leveraging team events and how can this understanding help to negotiate these constraints?

\section{Materials and Methods}

This study, which was conducted in a regional Australian area that hosts three national-level professional sport teams, forms part of a wider action research project on how team events can be leveraged for tourism benefits. The action research approach is focused on knowledge creation through experience with practitioners (Greenwood \& Levin, 2011). There are four cycles to the action research approach: plan (including research problem identification), act, observe, and reflect (Herr and Anderson, 2005). This study is based on the first cycle (plan) and part of the second cycle (act).

\subsection{Context}

The region that forms the research site consists of several cities with populations ranging from 60,000 to 200,000 . The regional area does not have a specifically dedicated tourism organisation. Instead, tourism is under the purview of each city's local government council. The cities are part of a regional tourism grouping that includes 13 local government councils from a broader geographic area; it is one of six destination networks within the state. Recently, a strategic development organisation was formulated to bring together the 13 councils from across the immediate area. The organisation was to set a broad strategic development plan, which included regional tourism.

In previous action research, semi-structured interviews and initial informal meetings have been used to understand interactions among key stakeholders before the intervention or action (Chalip et al., 2017; Taks et al., 2018). For our study, we conducted 25 semi-structured interviews with representatives of professional sport teams, leagues, and facilities; the different cities' events and tourism officials; and, local businesses. Participants were recruited 
through purposeful and snowball sampling (Patton, 2002). In addition to the interviews, a three-hour workshop with key stakeholders was undertaken. The workshop constituted the plan cycle of the action research process and the beginning of the act cycle.

\subsection{Data Collection}

Semi-structured interviews are beneficial for understanding a range of different viewpoints (Rubin \& Rubin, 2012). The 25 initial interviews ranged between 15 to 65 minutes and were used to comprehend the potential for and constraints to leveraging team events. The research questions were about event leveraging and team events more generally, and not specifically related to constraints. Questions included the following: How are sport events used to generate tourism? What has been done, if anything, to leverage events generally? Have there been any attempts to leverage team events specifically? How can events be leveraged for tourism? What benefits could team events provide the region from a tourism development perspective? Probing was also used to facilitate more in-depth discussion of the topic (Neuman, 2012).

Following the interviews, we conducted a three-hour workshop with eight stakeholders that included representatives from four of the 13 councils, the sports facility where the team events take place, and one of the sport teams. Although all 25 interviewees were invited, only eight could attend, and since the participants worked in a small regional area, no further identification can be provided to preserve participants' anonymity. As the workshop was designed to identify objectives and to begin planning the leveraging process, the information presented at the workshop related to team events, including crowd attendance and media reach as well as the reach of the leagues' teams and players. Workshops are used widely in the planning phase of action research projects to set objectives, design strategies, and delegate tasks (Chalip et al., 2017). 
The workshop consisted of a presentation of the findings from the initial interviews, including respondents' opinions on the potential of leveraging team events, and more information about team events. The presentation and discussion were followed by participant brainstorming about which sport team assets provided the best leveraging opportunities, which objectives could be achieved, what organisations needed in order to be involved in any leveraging initiatives, what resources were required, and what potential strategies might be employed. The concept of event leveraging (Chalip, 2004) was discussed with respondents in the interviews. It was explained that team events could provide opportunities through visitation and media attention that could lead to tourism benefits, if strategies were implemented to leverage the opportunities. While the term "event leveraging" was explained and used in the workshop by the researchers, it was not specifically used in the workshop by stakeholders. They did, however, discuss how team events could be used to produce both direct and indirect tourism benefits. In effect, the workshop was a discussion about event leveraging without always explicitly using the term.

After the workshop, we engaged in follow-up interviews with six key stakeholders to help guide the next stage of the leveraging process. Four of the stakeholders were present at the workshop, two were not. The follow-up interviews were used to clarify points made in the workshop and check on the progress of the group. The interviews and workshop were all recorded and transcribed verbatim.

\subsection{Data Analysis}

The data were analysed manually and coded using an inductive process to identify themes (Glaser \& Strauss, 1967). However, inductive analysis is rarely entirely inductive (Harding, 2018), and therefore, there was some deduction in the analytical process that was influenced by previous research. As Ragin (1994) argues, "it is impossible to research without some initial ideas" (p.47); therefore, the ideas generated from the literature review did have 
some influence on how the results were analysed.

The data analysis process involved reading the data repeatedly for familiarisation and then thematically coding the data, using open, axial, and selective coding (Corbin \& Strauss, 2008). Open coding was used to identify overarching themes and help focus on the core phenomena, which in this case comprised constraints to leveraging team events. Open coding was followed by axial coding, which identified the causal conditions of related concepts and categories as well as their context and conditions. Selective coding was used to refine the data into more unified 'core' categories and solidify the relationships among them (Corbin \& Strauss, 2008; Creswell, 2013). The data were coded by two researchers in an iterative process, with codes cross checked until agreement was reached. To ensure reliability, the researchers first read the codes independently to determine open codes (Strauss, 2008), and subsequently refined these into selective themes after discussion and agreement among the authors (Miles \& Huberman, 1994).

\section{Results}

Our results identified a series of constraints to leveraging team events, which led to the development of a process model (Figure 1). This diagrammatical representation of the leveraging constraints and negotiation process is conceptually based in research on processes, or how qualities of entities change over time (Langley, Smallman, Tsoukas, \& Van de Ven, 2013). Four initial constraints were identified: lack of collaboration, priorities and resources, perceived benefit radius, and perceived tourism potential. An intervention led to a shift in attitudes and opinions, but unclaimed responsibility was a constraint that prevented the realisation of the identified event leveraging opportunity. Within these overarching themes were several subthemes that further explain why these broader areas were constraints to leveraging team events. The quotes in the results section are used as examples indicative of research participants' responses. 


\section{[Figure 1 near here]}

\subsection{Lack of Collaboration}

As a constraint to leverage, lack of collaboration became manifest around convoluted organisational structures, lack of a unifying brand, and competition for the same target market.

\subsubsection{Convoluted Organisational Structures}

Respondents repeatedly stated that the convoluted structure of tourism in the region was a constraint to potential leveraging of team events: 'Being involved with the five destinations is difficult, it's frustrating .... The message is very confusing for the customers and the tourists' (T1). Respondents continually noted that being grouped with other areas was problematic: '[The region's major city] and the region should be a standalone area. We shouldn't be roped in to [the current grouping]' (T7).

The sharing of responsibility by many disjointed entities created a problem for efficiency, since each city had its own department responsible for tourism: 'I think that there's no one overarching entity or body, which is a big part of the problem. Every council will have their little tourism team' (T4). These disjointed structures led to a lack of unity, which prevented the progression of tourism development:

We have very passionate people all trying to articulate a way forward without a real strong framework. What it really needs is ... some sort of a body that incorporates all of those three different areas [state, regional, and local]. (T2)

Thus, the absence of a dedicated regional tourism organisation and the grouping of the respective cities' presence with other regions acted as a constraint to collaboration around leverage.

\subsubsection{Lack of Unifying Brand}

15

This is a pre-print of an article published by Taylor \& Francis in Sport Management Review on 29 Nov 2021, available online: https://doi.org/10.1080/14413523.2021.1989238 
The region's complex tourism structure eroded any unity among tourism bodies, in part because each city was seen as distinct and diverse: 'The reality is that it's difficult to bring together a region that's quite diverse, which is a strength, but in a marketing context, can be challenging' (T6). The lack of a unified brand, or more accurately, the presence of a diverse portfolio of brands, was challenging in a marketing context: 'I don't think there's a brand. I think there's a collection of thoughts, or a collection of images, or a collection of ideals... that different parts of the region project... there is not one overall brand' (T2). Clearly, this inability of the region to collaborate as a unified brand was a constraint to leveraging.

\subsubsection{Competition for the Same Target Market}

There was a suggestion that the lack of collaboration occurred because stakeholders from different cities in the region saw each other as competitors: 'I can't see [another stakeholder] working closely, hand in glove with [the city where the stadium is located] or hand in glove with [another of the regional cities]. At the moment, they see them as competition' (T14). Respondents saw the heavy reliance on one nearby capital city market as a key contributor to competition among cities within the region: 'They see themselves as competitors because about $80 \%$ of the tourism that goes to both areas comes out of [state capital city]' (T3). The competition for the same target markets, therefore, acted as a constraint to collaboration.

\subsection{Priorities and Resources}

The competing priorities of different stakeholders influenced how they could allocate resources, which constrained leverage and manifest as misalignment of existing priorities and insufficient temporal, financial and human resources. Specifically, results showed that team events did not align with stakeholders' existing priorities, which made it difficult to devote 
limited resources to leverage.

\subsubsection{Lack of Alignment with Existing Priorities}

The leveraging of team events did not align with the nascent strategic development organisation that was attempting to produce a broader economic strategy for the region that included tourism and events: 'The [regional organisation] is coordinating a number of projects across the region ... I'm not sure the sports one would sit up high on the list' (T8).

Even when the relevant stakeholders considered the leveraging of team events to be a worthwhile endeavour, without alignment, the leveraging would be very difficult to implement: 'Without it being written into the plans ... if you're trying to deliver on a plan, that's what you'll be working to and it doesn't leave much space for the other kind of stuff' (T17).

Lack of alignment was problematic for both tourism and sport stakeholders: 'I don't know if there is much of an importance placed on that [sport tourism initiatives] by the [sport] governing bodies' (T7). The teams also had to prioritise their day-to-day operations: 'To be honest, we've got a lot of other problems here ... to just make sure we're getting ready game to game. We don't have a big staff' (S2). Thus, stakeholders noted that their primary focus was on delivering existing strategies and day-to-day operations. Leveraging team events for tourism was, therefore, difficult if it was not explicitly included in such strategies.

\subsubsection{Resources}

Owing to the priorities of delivering on existing plans and day-to-day operations, stakeholders were challenged to find the required temporal, financial, and human resources needed to leverage team events for tourism. Some were responsible for delivering many major events and felt they could not devote time to leveraging team events: 'I just don't have the ability to put in as much effort and energy into every [team] game.' (S5). 
While stakeholders acknowledged that discussions to initiate tourism initiatives had taken place, finding temporal and financial resources was an obstacle to action: 'Time is one. Financial resources around putting money into common marketing is probably another [constraint]. Then the opportunity cost that the businesses might see with that' (T3).

Respondents also noted that a lack of human resources constrained their leveraging activity: 'It comes back to resources in terms of people. We are resource light at the moment, and that has a big effect on what we can do' (T19). There was an understanding that collaborations would be useful to overcome resourcing issues: 'It would be great to have the support of others because we don't have the resourcing to be able to do it wholly and solely' (T7).

Clearly, competing priorities placed demands on the availability of temporal, financial and human resources which constrained actors' respective abilities to exploit leveraging opportunities.

\subsection{Perceived Benefit Radius}

Respondents continually noted that if an event was staged in a particular city, then that city should benefit: 'From a stadium [perspective], it's very hard to take money from [the] City Council [where the stadium is located], and then go and try to pitch for money from other cities' (S5). Respondents also expressed that team events would primarily benefit the city in which they were located: 'It was all centred around [the stadium's host city] from our discussions last time. I mean, [our city], for example, people aren't necessarily going to come and stay here' (T19).

Equally, respondents believed that if they hosted an event, they would want the benefits to stay in their city:

If I said to my operators that we're going to do this marketing campaign with 
[another city in the region] and we're going to drive people to outside of our region, they'd be horrified, probably wouldn't buy into it. Ultimately, we want to keep people within our region. (T16)

Thus, cities were quite protective of their parochial interests: '[Cities are] looking out for their own patch, and that is where we've been divided in the past' (T1). Therefore, the perception that there would be an inequitable spread of team event benefits beyond the host city's immediate radius was a constraint to implementing leveraging initiatives.

\subsection{Perceived Tourism Potential}

Since respondents believed team events had only limited tourism potential, they did not view them as a significant leverageable resource. This belief was based on team events' perceived insignificance compared to other events, a level of complacency because of team events' regularity, target market incongruence, and a localised drawing radius.

\subsubsection{Comparative Insignificance to Other Events}

With professional sport teams heavily associated with the culture of the region, respondents assumed that team events did not attract tourism: 'I see them as part of the culture, I don't think [team events] bring a great deal of outside people' (T13). Respondents often noted that larger one-off events were used to benefit tourism in the region: 'Things like Matildas' [Australian national women's football team] games, Grand Finals ... they're the events that are really important to drive the tourism economy. [The events] are marketing the destination ... as a destination to come and visit' (S5). Importantly, this respondent highlights that regional benefits are not just in the direct effects of visitors for the event, but also the indirect benefits of destination marketing through event broadcasting.

While respondents consistently acknowledged that one-off events were tourism generators, they did not see team events in this light: 'The [sport team] game might bring in a 
couple thousand people from outside the region. But when you're looking at some of those international events, sometimes you bring in kind of 45 to $60 \%$ from outside the region' (S5). It is evident that an inability to see the tourism potential of team events, that was founded upon perceptions that they are relatively less significant generators of tourism than major oneoff events, acted as a constraint to leveraging team events.

\subsubsection{Complacency in Regularity}

The regularity of team events, which in this region, provide at least 12 home games a season, led some respondents to be somewhat complacent about their occurrence: 'I think where [our team] and [another team] are concerned, we're probably seen as being something of a, 'this is what always happens' (S4). There were a couple of respondents who went as far as to suggest that matches are not perceived as events: 'because I see it as just a regularly occurring thing. I see that as a fixture as such. A recurring fixture, but it's a fixture, like an attraction, but I don't see it as an event' (T6). Sport stakeholders were aware these perceptions had an impact on leveraging team events:

Well, I think that there would have to be an acceptance that a home and away game is going to be an event, right? I'm not sure they see [league] fixtures as events. And as a catalyst for them to be able to leverage that event (S6).

Complacency about team events was, therefore, a constraint to leverage.

\subsubsection{Target Market Incongruence}

Respondents expressed a widely held perception that the audience attracted by team events was incongruent with the region's primary tourism target markets: 'You're not really going to tack on a trip to the [wine region] as part of that game. It might be incongruent with [visitors'] expectations for their visit' (T9). Note that the respondent highlights that the region is widely known for food and wine. Respondents perceived attendees to team events would be 
uninterested in participating in such experiences. 'There's a belief that the [sport league] audience, for example, and I'm generalising, they're not interested in drinking wine. They're interested in drinking beer and bourbon' (T16). Significantly, respondents did not present nor refer to any actual market research to support this premise. Ultimately, these largely unfounded preconceptions about the consumer behaviours of team events' attendees have led to a perceived incongruence between the target markets of the event and the tourism product, which manifests as a constraint to leveraging team events.

4.4.3.1. Team Event Consumer Behaviours. Respondents who believed that team events might attract tourism also believed that those who travel to attend such events are solely interested in attending the game itself, and have little motivation for non-event touristic experiences: 'One of the problems with sport events is that people are so enthusiastic about the sport itself that the thought of visiting a wine destination is really not on their radar' (T16). Thus, the perception was that team events' attendees come for the game and return home immediately after: 'They come, they watch the match, and they go home. They don't come to the city and necessarily want to see the place' (T13). Therefore, respondents believe that the majority of team events' attendees would be solely concerned with attending the event and not engaging with other tourism attractions was a constraint to leveraging. Again, respondents did not present nor refer to any actual empirical market research to support these assumptions.

\subsubsection{Localised Drawing Radius}

Tourism stakeholders perceived that the majority of team events' attendees were drawn from the local region and were not tourists: 'A lot of [event attendees] are local. And we're not getting the overflow with accommodation. So, I don't really see that as a huge opportunity, from an immediate visitation perspective' (T6). This perception stems from an understanding that the supporters of visiting teams would not travel to watch their team: 
'Look, especially rugby league, it's a television sport. So, you follow Cronulla, for example, you probably wouldn't travel to [team host city] to come and watch it being played' (T16). The perception that most team events' attendees were drawn from the local region constituted a constraint to leveraging team events.

\subsection{Shift in Attitudes and Opinions}

In the process of conducting interviews and workshops, some participants began to consider the potential of team events as a leverageable resource for tourism. They were also given empirical data about team events, including in-person and media audiences as well as information about team event fixtures and the opponents of the three major sport teams playing in the region. This intervention led to a shift in opinions about team events as a leverageable resource for tourism.

After engaging in discussion, individuals who originally suggested that team events were not significant tourism generators expressed a different opinion: 'Maybe they're [the sport teams] sitting on a goldmine here.... What other market profile characteristics do their visitors have that can crossover with markets that would be interested in our product?' (T9). This shift in thinking was also demonstrated by respondents during the initial interviews: 'We're looking for things, and you've just kind of really opened my eyes up to something else that I've never, ever given a thought to' (S1). When respondents received more information about team events at the workshop, they began to consider them as a leverageable tourism resource: 'It's just never been recognised that we should be talking to a team ... from Melbourne. There's this huge [leveraging] opportunity for us [regional tourism stakeholders] all there, when they come up to play' (T8).

The shift in opinions about the potential for leveraging team events stemmed from stakeholders being able to see synergies between team events and tourism target markets: 'So, 
I can definitely see the potential, like social media collaborations with the [target market city] players that are coming to visit and play at [team host city]' (T17). Opinions on whether team events can be leveraged for tourism shifted as respondents learnt more about the actual nature of team events. Therefore, prior to intervention, a lack of awareness and inadequate information about team events, acted as a constraint to leverage.

\subsection{Unclaimed Responsibility}

Despite the changes in opinions about team events' potential as a tourism resource, the issue of responsibility remained a significant constraint. Specifically, the inability to identify who should lead and take responsibility for leveraging of team events inhibited action. The teams' and sport facilities' stakeholders believed it was the responsibility of tourism stakeholders: 'So, to me, tourism should be coming to us, assisting us to help them get more people come to town through football' (S2). There were respondents who suggested that those likely to receive a greater benefit, such as the city where the stadium is located, should lead the initiative:

Who should lead is a case of who would be benefiting the most. So, I've just felt like it was all centred around [the city where the stadium is located] ... just from the sporting teams they have, and the facilities they have and the recognition they have [they will benefit most]. (T19)

However, tourism stakeholders felt the sport leagues needed to initiate the process: 'I guess the key to the puzzle, and I know I keep coming back to this point, is the governing bodies of those codes, pointing to see the benefit, endorse it, and put some money into doing that' (T7).

It was evident in the post-workshop phase that no stakeholders considered their organisation to be the correct one to lead a leveraging initiative: 
When I started to look at it and go, okay, I think there's a really good opportunity there for the region, from the use of my time, and my investment, my local government area has probably not the highest opportunity.... I think we need an organisation at a higher level. (T8)

The absence of a single stakeholder willing to assume responsibility for leveraging team events represented an important constraint.

\section{Discussion}

Analysis of the findings revealed several constraints to leveraging team events. These included constraints in collaboration, priorities and resources, perceptions of limited tourism potential, and unclaimed responsibility. While an intervention informing participants about the potential of leveraging team events led to a shift in attitudes and opinions, unclaimed responsibility ultimately prevented the realisation of this leveraging potential. The process model developed from these findings offers an opportunity to understand constraints to leveraging team events over time, from before they are considered for leveraging, to when they are considered a legitimate leverageable resource.

To begin with, the complex tourism structure made collaboration difficult. As no single organisation had the capacity required to solely leverage team events, collaboration would be key to effectively leveraging the team event opportunities. Our findings are consistent with research identifying broader political decisions and priorities as constraints to leveraging (Bell \& Gallimore, 2015; Chalip et al., 2017; Chen \& Misener, 2019; Kennelly et al., 2017). However, our results suggest that political structures that may not affect leveraging directly, such as a Destination Management Organisation (DMO), can nevertheless constrain leveraging. The unwieldy tourism structure constrained the creation of common objectives (Kellett et al., 2008) and created unhealthy competition among stakeholders (Kennelly et al., 
2017). As this research considers events that were not being leveraged, it adds another layer of understanding to how political structures act as constraints in the pre-event leveraging phase. These findings support the argument that event leveraging requires a suitable alliance structure that facilitates stakeholder collaboration (Chalip et al., 2017; Wittmann, Hunt, \& Arnett, 2009).

Priorities across the different regional cities influenced the broader strategic event plans of each city and where they directed limited resources. As each city's event plans made no mention of team events, resourcing their leverage was not even considered. Alternatively, resources also shaped prioritisation in each city. As the cities had limited temporal, financial, and human resources, their priorities emphasised delivery of daily operations. Previous research has identified insufficient resources as a constraint to leverage (Bell \& Gallimore, 2015; Clark \& Misener, 2015; Hoskyn et al., 2018), as well as prioritising daily operations (Chalip et al., 2017; Kennelly et al., 2017).

In the initial phase of the research, respondents held a perception that team events had only marginal tourism potential, presenting a constraint to leverage. In effect, numerous tourism stakeholders did not consider these events capable of attracting significant visitor numbers or the type of visitor congruent with existing tourism target markets. Importantly, however, these perceptions were not based on objective data, but mere subjective assumptions. These assumptions were reinforced by a level of complacency related to the recurrent nature of these events - a characteristic that led some interviewees to not even consider them as events. In addition, respondents held a perception that team event benefits would be limited to the immediate host area, a perception consistent with results of event leveraging research in non-host areas (Beesley \& Chalip, 2011; Fairley, Cardillo, \& Filo, 2016; Kellett et al., 2008).

While our results are consistent with previous research, they also add another layer in 25 
that a lack of alignment with broader event strategies, and not just leveraging specifically, acts as a constraint to event leveraging. The results demonstrate that key regional actors routinely based strategic decision making on incomplete information. The strategic plans of organisations and the assumptions that underpin those plans help determine priorities (Poister $\&$ Streib, 2005) and resource allocations (Steiss, 1985). This is a significant and new perspective for event leveraging, as previous research has considered events that have already been identified for leveraging initiatives, and therefore, form part of event strategies. This study extends this understanding and suggests that event stakeholders need to think more broadly about strategic control and, in particular, feedforward control mechanisms like premise control (Harrison \& St. John, 2014) to feed into broader regional strategies and the resource prioritisation decisions required for leveraging. Identification of leverageable events as part of broader regional tourism strategies and the allocation of resources for leverage should, therefore, be founded upon evidence-based decision-making, rather than merely subjective and potentially flawed assumptions. This suggests that the establishment of accurate strategic control mechanisms may be a prerequisite for including team events as part of a host region's event portfolio and achieving any useful leverage of the opportunities they present.

While opinions relating to leveraging actions have been identified as constraints in previous studies (Chalip et al., 2017; Chen \& Misener, 2019; Taks et al., 2014; 2018), our results reveal that opinions regarding the usefulness of the event as a leverageable resource are a significant constraint. This is a key finding because it reveals that prevailing opinions of how different types of events produce leveraging opportunities can actually constrain leverage. This may be linked to organisational culture issues as identified by Chalip et al. (2017), as well as broader socio-cultural expectations, such as stereotypes about particular sport fans behaving in certain ways, for instance, preferring beer and bourbon to wine. It also 
demonstrates that those with the most to gain may not recognise team events as a significant leverageable resource. Our research thus builds on Mason and Duquette's (2008) conclusion that team events remain an untapped resource.

However, an intervention which included information about the events' audiences led to consideration of team events as a useful leverageable resource. As participants received more information about the team events' attendances and media audiences, and considered the related leveraging opportunities more deeply, opinions began to shift. An understanding that, over the course of the season, team events could attract a significant number of visitors lessened the perception of limited tourism potential. Confronted with actual data, stakeholders began to see how they could leverage the teams' respective media profiles to reach audiences they previously had difficulty reaching. A gathering (e.g. workshop) that facilitated discussion, and presented team event information was, therefore, an important intervention that led to the shift in opinions of stakeholders to perceive team events as a leverageable asset to help achieve common objectives.

Ultimately, however, unclaimed responsibility for leading leveraging initiatives remained a constraint to further leveraging action. Several event leveraging studies have found an inability to identify responsibility for leveraging actions to be a constraint (Misener, 2015; Kennelly et al., 2017; Taks et al, 2014; 2018). The assumption that 'someone else' was responsible (Misener, 2015) and the lack of a champion (Kennelly et al., 2017) were also contributors to unclaimed responsibility in this study. However, the findings here reveal another aspect that has not been considered in previous event leveraging research, which is that organisations that have the ability to coordinate the different leveraging stakeholders need to be active. In this case, state tourism organisations and the respective league governing bodies were perceived to be key in coordinating the different actors required to leverage team events. In addition, the regularity of team events was shown to create a lack of urgency, in 
contrast to one-off or periodic events, which have definite deadlines for when initiatives need to be implemented. Therefore, this study's results indicate that leveraging of regular events may be consistently postponed, unless responsibility for leveraging is identified by an actor with the ability to coordinate leveraging stakeholders.

Understanding who will lead and be responsible for leveraging is, therefore, fundamental for leveraging team events. Including team events as part of a host's event portfolio could alleviate this constraint, legitimise team events as leverageable, and help motivate the involvement and coordination of the different stakeholders involved. If a specific organisation is responsible for overseeing the event portfolio, as is the case in London, Canada (Clark \& Misener, 2015), Auckland, New Zealand (Ziakas, 2019), and the regional Australian city in Kelly and Fairley's (2018) work, such an organisation could then conceivably assume responsibility for leading the leverage of team events. The organisation could also establish the necessary strategic controls and facilitate collaboration among sport and tourism stakeholders. The absence of such an organisation in this case was a major constraint to leveraging team events. While event portfolios are viewed as a key part of event leveraging (Chalip, 2004), previous research has not considered how the absence of a particular event category, in this case, team events, from a portfolio can constrain leveraging efficacy overall.

\section{Conclusion}

This study found several constraints to leveraging team events and developed a process model to help negotiate these. The model highlights that constraints relating to the collaborative structure and assumptions about events can act as significant impediments to event leveraging. These may be negotiated with an intervention that leads to a shift in attitudes and opinions. However, identifying responsibility for leverage is a key antecedent to ultimately realising leveraging potential. 
The findings support the conclusion that knowledge of the event itself is an important factor in effective leveraging and certainly cannot be taken for granted (Chalip et al., 2017). It follows that, as a constraint to leverage, absence of knowledge can be reduced through interventions whereby stakeholders are informed about the leveraging potentials of specific events, in this case, team events. Therefore, this research advances the theoretical understanding of event leverage by proposing that societal conceptualisations of events significantly impact how events are used, or not, for achieving targeted host community outcomes. In other words, how a host community perceives an event will greatly influence whether the event is leveraged, or not, for achieving host benefits.

Therefore, building on Beesley and Chalip's (2011) notion that event leveraging is viewed differently among different cultures (Beesley \& Chalip, 2011), this research demonstrates that also within communities, diverse perceptions exist around the types of events that are leverageable. This highlights the need for event stakeholders to understand community perceptions of their event. Such understanding becomes a platform upon which to provide interventions in the form of evidence-based educational workshops and community outreach programs aimed at shifting community attitudes and building understanding around the utility of events for producing desired benefits.

\section{Managerial Implications}

Cities hoping to use team events as a means to achieve various objectives need to first recognise that these events are in fact leverageable. Then, structures that facilitate effective strategic controls and collaboration among key stakeholders need to be established. Establishing such structures may require higher authorities, such as state tourism bodies or sport leagues, to initiate collaboration among stakeholders. Some stakeholders may not consider team events to be a leverageable resource, thereby establishing a requirement for strategic controls such as detailed empirical data about attendees, target markets, and media 
reach, as well as - in the case of team events - information on opposing teams' audiences, media reach, and the athletes competing.

Some stakeholders may not be able to allocate resources to leveraging if team events are not included in broader regional event strategies. Including team events as part of a city's event portfolio legitimises their place in the city's event plans and broader marketing mix, and enables allocation of resources to leveraging. The constraint of unclaimed responsibility for leveraging team events may also be overcome through inclusion of team events in an event portfolio, as the collaborative alliance overseeing the portfolio can lead, or at least delegate, responsibility and resource coordination for leveraging action.

\section{Limitations and Future Research}

This study had some limitations. The geographic area where the study took place, as well as its tourism structure, may not be generalisable. In addition, the researchers were unable to connect with the state tourism body, which is considered a crucial stakeholder for leveraging team events. While the majority of stakeholders signalled their intention to take part in the workshop, some key stakeholders were absent when the workshop took place. Future research should look at developing and testing strategies designed to reduce constraints in leveraging team events. A longitudinal study may also more accurately monitor how stakeholders engage with team events over a sustained period. Understanding how team events may be effectively integrated into a region's event portfolio is another area that warrants further investigation to overcome the constraints of team event leveraging. This study was specifically focused on leveraging team events for tourism benefits, however, there is significant potential for other benefits from leveraging team events. Further research should consider this, for instance leveraging team events for social objectives, such as increased sport participation outcomes. 


\section{References}

Argyris, C. and Schon, D. (1978). Organisational learning: A theory of action perspective. New York: Addison-Wesley.

Beesley, L. G., \& Chalip, L. (2011). Seeking (and not seeking) to leverage mega-sport events in non-host destinations: The case of Shanghai and the Beijing Olympics. Journal of Sport \& Tourism, 16(4), 323-344.

Bell, B., \& Gallimore, K. (2015). Embracing the games? Leverage and legacy of London 2012 Olympics at the sub-regional level by means of strategic partnerships. Leisure Studies, 34(6), 720-741.

Chalip, L. (2004). Beyond impact: A general model for sport event leverage. In B. W. Ritchie \& D. Adair (Eds.), Sport tourism: Interrelationships, impacts and issues (pp. 226252). Clevedon, England: Channel View Publications.

Chalip, L. (2017). Trading legacy for leverage. In I. Brittain, J. Bocarro, T. Byers, \& K. Swart (Eds.), Legacies and mega events: Fact or fairy tales? (pp. 45-62). London: Routledge.

Chalip, L., Green, B. C., Taks, M., \& Misener, L. (2017). Creating sport participation from sport events: Making it happen. International Journal of Sport Policy and Politics, 9(2), 257-276.

Chen, S., \& Misener, L. (2019). Event leveraging in a nonhost region: Challenges and Opportunities. Journal of Sport Management(00), 1-14.

Clark, R., \& Misener, L. (2015). Understanding urban development through a sport events portfolio: A case study of London, Ontario. Journal of Sport management, 29(1), 1126. 
Corbin, J., \& Strauss, A. (2008). Basics of qualitative research: Techniques and procedures for developing grounded theory (3rd ed.). Thousand Oaks: Sage.

Creswell, J. W. (2013). Qualitative inquiry and research design : Choosing among five approaches (3rd ed.). Thousand Oaks: Sage.

Duignan, M., Kirby, S., O’Brien, D., \& Everett, S. (2018). From 'clone towns to 'slow towns': Examining festival legacies. Journal of Place Management and Development. 11(3), $350-366$.

Duignan, M., Down, S., \& O'Brien, D. (2020). Entrepreneurial leveraging in liminoidal Olympic transit zones. Annals of Tourism Research, 80, 102774.

Fairley, S., Cardillo, M. L., \& Filo, K. (2016). Engaging volunteers from regional communities: non-host city resident perceptions towards a mega-event and the opportunity to volunteer. Event Management, 20(3), 433-447.

Fairley, S., \& Gammon, S. (2005). Something lived, something learned: Nostalgia's expanding role in sport tourism. Sport in Society, 8(2), 182-197.

Gibson, H. (1998). Sport tourism: A critical analysis of research. Sport Management Review, $1(1), 45-76$.

Gibson, H., Willming, C., \& Holdnak, A. (2002). "We're Gators... not just Gator fans": Serious leisure and University of Florida football. Journal of Leisure Research, 34(4), $397-425$.

Gibson, H. J., Willming, C., \& Holdnak, A. (2003). Small-scale event sport tourism: Fans as tourists. Tourism Management, 24(2), 181-190.

Glaser, B. G., \& Strauss, A. L. (1967). The Discovery of grounded theory: Strategies for qualitative research. Chicago: Aldline. 
Greenwood, D. J., \& Levin, M. (2011). Introduction to action research: Social research for social change. Thousand Oaks: SAGE publications.

Grix, J. (2012). 'Image' leveraging and sports mega-events: Germany and the 2006 FIFA World Cup. Journal of Sport \& Tourism, 17(4), 289-312.

Harding, J. (2018). Qualitative data analysis from start to finish. London: SAGE

Harrison, J. S., \& St. John, C. H. (2014). Foundations in strategic management (6th ed.). Mason, OH: Thomson South-Western.

Herr, K., \& Anderson, G. L. (2005). The action research dissertation: A guide for students and faculty. Thousand Oaks: Sage.

Higham, J. (1999). Commentary-sport as an avenue of tourism development: An analysis of the positive and negative impacts of sport tourism. Current Issues in Tourism, 2(1), $82-90$.

Higham, J., \& Hinch, T. D. (2003). Sport, space, and time: Effects of the Otago Highlanders franchise on tourism. Journal of Sport Management, 17(3), 235-257.

Hinch, T., Jackson, E. L., Hudson, S., \& Walker, G. (2005). Leisure constraint theory and sport tourism. Sport in Society, 8(2), 142-163.

Hoskyn, K., Dickson, G., \& Sotiriadou, P. (2018). Leveraging medium-sized sport events to attract club participants. Marketing Intelligence \& Planning, 36(2), 199-212.

Jones, I. (2008). Sport fans and spectators as sport tourists. Journal of Sport \& Tourism, 13(3), 161-164.

Karadakis, K., Kaplanidou, K., \& Karlis, G. (2010). Event leveraging of mega sport events: a SWOT analysis approach. International Journal of Event and Festival Management, 1(3), 170-185. 
Kellett, P., Hede, A.-M., \& Chalip, L. (2008). Social policy for sport events: Leveraging (relationships with) teams from other nations for community benefit. European Sport Management Quarterly, 8(2), 101-121.

Kelly, D. M., \& Fairley, S. (2018). The utility of relationships in the creation and maintenance of an event portfolio. Marketing Intelligence \& Planning, 36(2), 260-275.

Kelly, D. M., Fairley, S., \& O'Brien, D. (2019). It was never ours: Formalised event hosting rights and leverage. Tourism Management, 73, 123-133.

Kennelly, M., Corbett, H., \& Toohey, K. (2017). Leveraging ambitions and barriers: Glasgow universities and the 2014 Commonwealth Games. Marketing Intelligence \& Planning, $35(6), 822-838$.

Lamont, M., Kennelly, M., \& Wilson, E. (2012). Competing priorities as constraints in event travel careers. Tourism Management, 33(5), 1068-1079.

Langley, A. N., Smallman, C., Tsoukas, H., \& Van de Ven, A. H. (2013). Process studies of change in organization and management: Unveiling temporality, activity, and flow. Academy of Management Journal, 56(1), 1-13.

Mason, D., \& Duquette, G. (2008). Exploring the relationship between local hockey franchises and tourism development. Tourism Management, 29(6), 1157-1165.

Mhanna, R., Blake, A., \& Jones, I. (2017). Challenges facing immediate tourism leveraging: evidence from the London 2012 Olympic Games. Managing Sport and Leisure, 22(2), 147-165.

Miles, M.B. and Huberman, A.M. (1994), Qualitative data analysis: An expanded sourcebook, 2nd ed. Thousand Oaks: Sage. 
Misener, L. (2015). Sports governance, development and corporate responsibility. International Journal of Sport Policy and Politics, 6(3), 550-552.

Misener, L., Taks, M., Chalip, L., \& Green, B. C. (2015). The elusive "trickle-down effect" of sport events: Assumptions and missed opportunities. Managing Sport and Leisure, $20(2), 135-156$.

Neuman, W. L. (2012). Basics of social research: Qualitative and quantitative approaches (3rd ed.). Upper Saddle River, NJ: Pearson.

Nieboer, N. (2011). Strategic planning process models: A step further. Property Management, 29 (40), 371-382

O'Brien, D. (2006). Event business leveraging The Sydney 2000 Olympic Games. Annals of Tourism Research, 33(1), 240-261.

O'Brien, D. (2007). Points of leverage: Maximizing host community benefit from a regional surfing festival. European Sport Management Quarterly, 7(2), 141-165.

O'Brien, D., \& Chalip, L. (2007). Executive training exercise in sport event leverage. International Journal of Culture, Tourism and Hospitality Research, 1(4), 296-304.

O’Brien, D., \& Chalip, L. (2007). Sport events and strategic leveraging: Pushing towards the triple bottom line. In A. G. Woodside \& D. Martin (Eds.), Tourism management: Analysis, behaviour, and strategy (pp. 318-338). Wallingford: CAB International.

O’Brien, D., \& Gardiner, S. (2006). Creating sustainable mega event impacts: Networking and relationship development through pre-event training. Sport Management Review, 9(1), 25-47.

O’Brien, D., Parent, M., Ferkins, L., \& Gowthorp, L. (2019). Strategic management in sport. London: Routledge. 
Patton, M. Q. (2002). Qualitative research and evaluation methods (3rd ed.). Thousand Oaks: Sage.

Poister, T. H., \& Streib, G. (2005). Elements of strategic planning and management in municipal government: Status after two decades. Public Administration Review, 65(1), 45-56.

Ragin, C. (1994) Constructing social research: The unity and diversity of method. Newbury Park, CA: Pine Forge Press.

Rubin, H. J., \& Rubin, I. S. (2012). Qualitative interviewing: The art of hearing data (2nd ed.). Thousand Oaks: Sage.

Sparvero, E., \& Chalip, L. (2007). Professional teams as leverageable assets: Strategic creation of community value. Sport Management Review, 10(1), 1-30.

Steiss, A. W. (1985). Strategic management and organizational decision making. Lexington: D.C. Heath.

Taks, M., Green, B. C., Misener, L., \& Chalip, L. (2014). Evaluating sport development outcomes: the case of a medium-sized international sport event. European Sport Management Quarterly, 14(3), 213-237.

Taks, M., Green, B. C., Misener, L., \& Chalip, L. (2018). Sport participation from sport events: Why it doesn't happen? Marketing Intelligence \& Planning, 36(2), 185-198.

Wittmann, C. M., Hunt, S. D., \& Arnett, D. B. (2009). Explaining alliance success: Competences, resources, relational factors, and resource-advantage theory. Industrial Marketing Management, 38(7), 743-756.

Xifra, J. (2009). Building sport countries' overseas identity and reputation: A case study of public paradiplomacy. American Behavioral Scientist, 53(4), 504-515. 
Ziakas, V. (2019). Issues, patterns and strategies in the development of event portfolios:

Configuring models, design and policy. Journal of Policy Research in Tourism, Leisure and Events, 11(1), 121-158. 